\title{
Centromere-associated repeat arrays on Trypanosoma brucei chromosomes are much more extensive than predicted
}

\author{
Maria C Echeverry ${ }^{1,2}$, Christopher Bot ${ }^{1}$, Samson O Obado ${ }^{1,3}$, Martin C Taylor $^{1}$ and John M Kelly ${ }^{1 *}$
}

\begin{abstract}
Background: African trypanosomes belong to a eukaryotic lineage which displays many unusual genetic features. The mechanisms of chromosome segregation in these diploid protozoan parasites are poorly understood. Centromeres in Trypanosoma brucei have been localised to chromosomal regions that contain an array of $\sim 147 \mathrm{bp}$ AT-rich tandem repeats. Initial estimates from the genome sequencing project suggested that these arrays ranged from 2 - $8 \mathrm{~kb}$. In this paper, we show that the centromeric repeat regions are much more extensive.

Results: We used a long-range restriction endonuclease mapping approach to more accurately define the sizes of the centromeric repeat arrays on the $8 \mathrm{~T}$. brucei chromosomes where unambiguous assembly data were available. The results indicate that the sizes of the arrays on different chromosomes vary from 20 to $120 \mathrm{~kb}$. In addition, we found instances of length heterogeneity between chromosome homologues. For example, values of 20 and $65 \mathrm{~kb}$ were obtained for the arrays on chromosome 1, and 50 and $75 \mathrm{~kb}$ for chromosome 5.

Conclusions: Our results show that centromeric repeat arrays on T. brucei chromosomes are more similar in size to those of higher eukaryotes than previously suspected. This information provides a firmer framework for investigating aspects of chromosome segregation and will allow epigenetic features associated with the process to be more accurately mapped.
\end{abstract}

\section{Background}

Centromeres are the chromosomal loci that facilitate segregation in most eukaryotes. They are the site of assembly of the kinetochore, the nucleoprotein complex which anchors the microtubule spindles that separate sister chromatids and mediate their movement to the daughter nuclei. Most centromeres are "regional" and encompass large sections of DNA, spanning 0.06 - 5 $\mathrm{Mb}$, in species as diverse as plants, insects and mammals [1-3]. Centromeric DNA is typically comprised of arrays of highly repeated sequences, interrupted by transposable elements $[4,5]$. The repeats are generally restricted to centromeric regions and are often in the size range 150 - $180 \mathrm{bp}$. This length is similar to that of nucleosomes, a property that may be of functional significance [6]. Although many features of centromeric

\footnotetext{
* Correspondence: john.kelly@lshtm.ac.uk

'Department of Infectious and Tropical Diseases, London School of Hygiene and Tropical Medicine, Keppel Street, London WC1E 7HT, UK Full list of author information is available at the end of the article
}

DNA are widespread, there is little sequence conservation, even between closely related species [7], and most evidence suggests that centromeres are determined epigenetically $[8,9]$.

In human chromosomes, centromeres have a conserved core of $\alpha$-satellite repeats $(\sim 170 \mathrm{bp})$ stretching over several megabases, which is flanked by extensive regions that contain multiple retrotransposon insertions [4]. In eukaryotic microorganisms, centromeres can also encompass large regions of chromosomal DNA. Those of Schizosaccharomyces pombe for example, range from $35-110 \mathrm{~kb}[10]$ and are organised as chromosome-specific core elements, flanked by inverted arrays of $3-7$ $\mathrm{kb}$. These in turn are flanked by more extensive outer repeats. Unusually in Saccharomyces cerevisiae, the regions that specify kinetochore assembly are restricted to single 125 bp elements termed "point" centromeres [11]. Some organisms, such as Caenorhabditis elegans, have holocentric chromosomes that lack specific
C Biomed Central 
centromeres [12]. In these instances, microtubules bind along the entire length of the chromosome.

Protozoan parasites of the Trypanosoma brucei species complex are insect-transmitted pathogens that are of major medical and veterinary importance throughout sub-Saharan Africa. They belong to the Excavata, a eukaryotic lineage which includes the other trypanosomatid parasites Trypanosoma cruzi and Leishmania species. Several features of gene organisation and expression in these organisms are unusual. Protein coding genes lack conventional RNA polymerase II (pol II) promoters [13] and are organised in long co-directional clusters which can stretch for tens to hundreds of kilobases [14]. Transcription is polycistronic, and processing involves a trans-splicing mechanism in which all mRNAs are modified post-transcriptionally by the addition of a 39-nucleotide spliced leader to their 5 '-ends. $T$. bruce $i$ has a haploid genome content of $35 \mathrm{Mb}$, with 11 megabase pair chromosomes (0.9 - 5.7 Mb). Unusually, chromosome homologues can vary significantly in size [14]. In addition, this parasite also contains two classes of atypical nuclear chromosomes; the intermediate-size chromosomes $(300-900 \mathrm{~kb})$ that contain some variant surface glycoprotein (VSG) genes, but no house-keeping genes, and the minichromosomes $(50-100 \mathrm{~kb})$, which appear to act as a reservoir of VSG sequences [15].

The T. brucei genome project was completed in 2005 [14]. However, sequence elements characteristic of centromeric DNA in other eukaryotes were not described. Furthermore, candidates for the 'core' centromeric proteins and most of the other factors involved in kinetochore assembly could not be identified $[14,16]$. This includes the variant histone $\mathrm{CenH} 3$, which specifies centromere location in eukaryotes and was thought to be ubiquitous [17]. The first evidence on the nature and location of centromeric DNA in T. brucei came from a biochemical mapping approach based on etoposidemediated topoisomerase-II cleavage $[18,19]$. Topoisomerase-II has a major regulatory role in chromosome segregation and accumulates at centromeres during late metaphase, where it resolves the catenated DNA strands that provide the final structural link between sister kinetochores [20,21]. This process requires double stranded DNA cleavage, passage of the uncut duplex through the gap and re-ligation to repair the break. Etoposide inhibits this re-ligation step leading to lesions in chromosomal DNA at sites of topoisomerase-II activity. In human chromosomes, etoposide-mediated cleavage sites occur within the $\alpha$-satellite repeats that constitute centromeric DNA [22,23]. In both T. cruzi [24] and Plasmodium $[25,26]$, these sites have been delineated to chromosomal loci that confer mitotic stability. In Toxoplasma gondii, they co-locate with the binding sites of the centromeric histone CenH3 [27].
Using the etoposide mapping method, we identified the location of putative centromeric domains on the 8 T. brucei chromosomes that had been fully assembled [18]. These loci, which occur once per chromosome, encompass regions between directional gene clusters that contain transposable elements and an array of AT-rich repeats predicted to extend between 2 and 8 $\mathrm{kb}$. The tandem repeats are arranged in units of $\sim 147$ bp and share intra-chromosomal identities ranging from $50 \%$ to more than $90 \%$. The units have a complex structure made up of degenerate sub-repeats of $\sim 48$ and $\sim 30$ bp (for a more detailed description of their make-up, see reference [18]). We also noted that the repeat arrays were located adjacent to ribosomal RNA genes on 5 of the chromosomes, although the significance of this is unknown. The intermediate and minichromosomes did not exhibit site-specific topoisomerase-II activity, suggesting that their segregation might involve a centromere-independent mechanism, a finding consistent with the "lateral-stacking" model [15].

In the initial analysis of the T. brucei centromeric domains, we identified discrepancies between the published sequence data of two chromosomes and our preliminary long range restriction mapping [18]. We also found evidence of heterogeneity in the extent of these regions between chromosome homologues. However, it was unclear whether the differences arose from an under-estimation of the copy number of the tandem repeats, whether they were due to the gaps in the assembly of the adjacent regions, or whether this underestimation of size was also the case with other T. brucei chromosomes. Here, we show that the centromeric repeats in T. brucei chromosomes are present at much higher copy number than predicted, with an organisation that is more typical of centromeric domains in higher eukaryotes than realised. These data provide a more complete model for $T$. brucei chromosome structure, an improved basis for investigating the mechanisms of segregation, and will enable more detailed functional mapping of this crucial chromosomal region to be undertaken.

\section{Methods}

\section{Parasites and DNA preparation}

T. brucei procyclic forms (genome project strain TREU 927/4) were grown in SDM-79 medium [28] with $10 \%$ heat-inactivated fetal bovine serum at $28^{\circ} \mathrm{C}$. For preparation of intact chromosomal DNA, the agarose embedding technique was used [29]. $10^{8}$ procyclics were immobilized in $1 \%$ low melting-point agarose blocks and incubated at $48^{\circ} \mathrm{C}$ for 48 hours in proteinase $\mathrm{K} / \mathrm{sar}$ cosyl buffer. Genomic DNA was extracted using the phenol-chloroform method [30]. 
In situ digestion and electrophoretic resolution

Prior to incubation with restriction endonucleases, agarose blocks were washed 3 times for 1 hour at $48^{\circ} \mathrm{C}$ in 50 volumes of TE buffer $(10 \mathrm{mM}$ Tris- $\mathrm{HCl}, 1 \mathrm{mM}$ EDTA, pH 7.4) containing $40 \mu \mathrm{g} \mathrm{ml}^{-1}$ phenylmethanesulphonylfluoride to inactivate proteinase K. After a minimum of 2 hours equilibration with the respective restriction enzyme buffer, blocks were incubated with restriction enzymes for 48 hours at $37^{\circ} \mathrm{C}$. Fresh enzyme (5 - 10 units) was added at the 24 and 36 hour time points. The digested DNA was resolved by a CHEF (contour-clamped homogenous electric field) Mapper System (Bio-Rad) (typically quarter of a block was used per lane) using an auto-algorithm set to the designated molecular mass range. For resolution of DNA fragments less than $20 \mathrm{~kb}$, genomic DNA (5 - $10 \mu \mathrm{g})$ was digested for 3 hours and fractionated on $0.5 \%$ agarose gels using standard electrophoresis techniques. As molecular size markers, a combination of Bio-Rad CHEF DNA standards 8 - $48 \mathrm{~kb}$, lambda ladder $50-1000 \mathrm{~kb}$ and S. cerevisiae chromosomes from 225 - 2,200 kb were used. Southern blotting was performed using standard procedures as outlined previously [24].

\section{Results}

The locations of centromeric regions on T. brucei chromosomes 9 - 11 have been predicted from etoposidemediated mapping [19], however incomplete assembly of the corresponding regions negates accurate longrange restriction mapping. For this study, we therefore focused on T. brucei chromosomes 1 - 8 (Table 1). As a first step, we generated in silico restriction digestion maps, based on the sequences available in GeneDB (Additional file 1). Our aim was to identify enzymes which cut proximal to the ends of centromeric arrays [31] and allowed the generation of paired overlapping fragments containing the repeat arrays, which could then be sized following electrophoresis. We also sought to identify sequences located adjacent to the centromeric region to act as single copy probes. Mostly these were open reading frames (Additional file 1). The abundance of high copy number elements adjacent to the tandem repeats (typically retrotransposons and ribosomal RNA genes) was in some cases a limiting factor. Below we describe our approach to delineating the repeat arrays, using chromosomes 3,5 and 7 as examples. The complete data set, including full analysis of the other chromosomes, is shown in Additional files 2 and 3, and summarised in Table 1. Fragment sizes greater than $20 \mathrm{~kb}$ were estimated to the nearest $5 \mathrm{~kb}$.

\section{Chromosome 3}

Sequence data (GeneDB) had suggested that the repeat array on this chromosome could be isolated on a Not I fragment of $153 \mathrm{~kb}$. However, Southern analysis of Not I digested DNA, following fractionation by CHEF (Figure 1), revealed fragments of 220 and $225 \mathrm{~kb}$. This indicated the presence of $\sim 70 \mathrm{~kb}$ of additional DNA in this region and was consistent with heterogeneity between chromosome homologues. Two enzymes, SgrA I and Sfi I, were used for analysis of sequences upstream of the array. Single fragments were identified, which were in both cases slightly larger $(5 / 10 \mathrm{~kb})$ than predicted. To investigate the downstream region, we used $B a m \mathrm{H}$, which cuts within $2 \mathrm{~kb}$ of the repeat array and was predicted to liberate a fragment of $104 \mathrm{~kb}$. This produced two fragments on the autoradiograph, one of which was 10 $15 \mathrm{~kb}$ shorter than expected. These small differences, which may arise from the haploid mosaic nature of the genome sequence [14], cannot account for the larger than predicted size of the Not I fragment. The data therefore suggest that the centromeric repeat region on

Table 1 Inferred sizes of the centromeric tandem arrays on chromosomes 1 - 8.

\begin{tabular}{|c|c|c|c|c|}
\hline Chr no. & Chr size & $\begin{array}{l}\text { Size of centromeric } \\
\text { repeat array (GeneDB) }\end{array}$ & $\begin{array}{l}\text { Size inferred } \\
\text { from mapping }\end{array}$ & Artemis coordinates $^{b}$ \\
\hline 1 & 1.15/1.2 Mb & $5.2 \mathrm{~kb}$ & $20 \& 65 \mathrm{~kb}$ & $780179 \ldots .785416$ \\
\hline 2 & $1.3 \mathrm{Mb}$ & $8.2 \mathrm{~kb}$ & $30 \& 55 \mathrm{~kb}$ & 290326...298494 \\
\hline 3 & 1.8/2.0 Mb & $7.8 \mathrm{~kb}$ & $75 \& 80 \mathrm{~kb}$ & $884223 \ldots . .891983$ \\
\hline 4 & $1.9 / 2.0 \mathrm{Mb}$ & $3.6 \mathrm{~kb}$ & 70 kb & $954159 \ldots . .957772$ \\
\hline 5 & $2.0 \mathrm{Mb}$ & $1.9 \mathrm{~kb}$ & $50 \& 75 \mathrm{~kb}$ & 197919...199815 \\
\hline 6 & $2.0 / 2.5 \mathrm{Mb}$ & $5.4 \mathrm{~kb}$ & $55 \mathrm{~kb}$ & $59066 \ldots . .64433$ \\
\hline 7 & $2.7 \mathrm{Mb}$ & $3.1 \mathrm{~kb}$ & $100 \& 120 \mathrm{~kb}$ & 1936979....1940105 \\
\hline 8 & $2.7 / 2.9 \mathrm{Mb}$ & unknown ${ }^{c}$ & $100 \mathrm{~kb}$ & $2233201 \ldots . .2233419^{d}$ \\
\hline
\end{tabular}

The approaches used to infer the extent of each of the centromeric repeat arrays are outlined in the text (chromosomes 3, 5 and 7) and Additional file 2 (chromosomes 1, 2, 4, 6 and 8).

${ }^{\mathrm{b}}$ The coordinates were defined using the Tandem Repeats Finder Program [31]. The positions in the sequence where the programme detected the first and the last motif were defined as the beginning and as the end of the centromeric repeat region. Coordinates are from T. brucei 927 version 4 (GeneDB). 'The size of the centromeric repeat array is not defined in GeneDB due to incomplete gap closure.

${ }^{\mathrm{d}}$ The coordinates correspond to blast hits with chromosome 4 centromeric repeat sequences. These occur adjacent to a gap of undetermined size. 


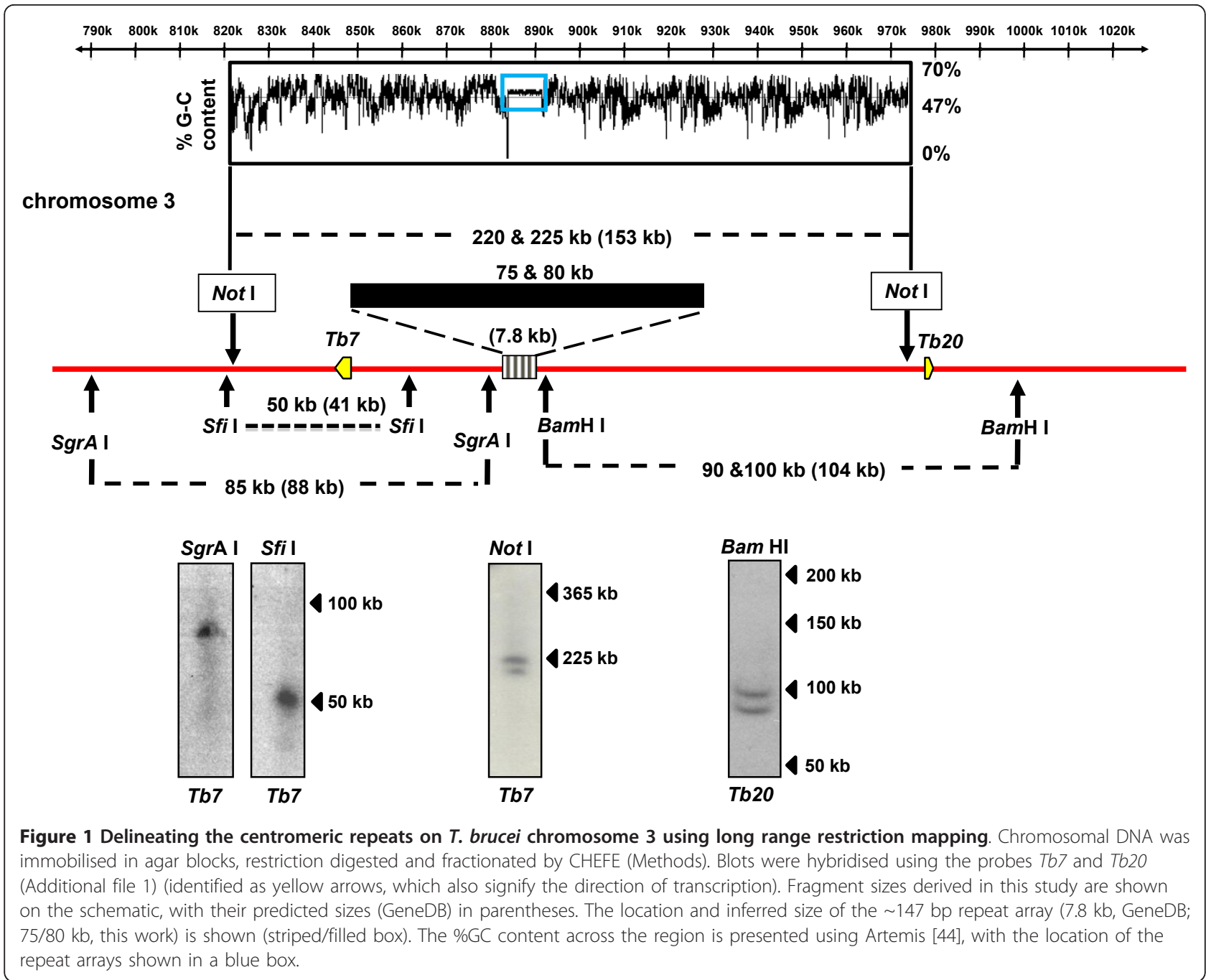

this chromosome is considerably more extensive than expected and that it may be of slightly different length on each homologue. This latter inference is not unambiguous, because of the heterogeneity in the flanking $B a m \mathrm{H}$ I fragment. A paucity of single copy probes and convenient restriction sites in this region limited our ability to address this further.

\section{Chromosome 5}

In accordance with genome sequence data, digestion with Not I should have generated a $31 \mathrm{~kb}$ fragment that contains the centromeric repeat array from chromosome 5. However, Southern hybridisation identified fragments of 80 and $105 \mathrm{~kb}$ (Figure 2), implying heterogeneity between homologues and the presence of 50 and $75 \mathrm{~kb}$ of additional DNA in the centromeric region. Analysis of an $M f e$ I digest (Figure 2) indicated that this did not arise from additional sequences in the immediate downstream region. When the upstream region was analysed following an SgrA I digest, two fragments were identified, which were 5 and $20 \mathrm{~kb}$ larger than predicted. Therefore, some of the size heterogeneity observed with the Not I digest could be due to additional sequences in this upstream region. However, the vast majority of the additional sequence in the Not I fragment, must arise from DNA within, or immediately adjacent to the repeat array (Figure 2).

\section{Chromosome 7}

The repeat array on this chromosome was predicted to be located on a Swa I fragment of $112 \mathrm{~kb}$. Southern analysis however, identified a doublet of 210 and $230 \mathrm{~kb}$ (Figure 3). Digestion with Ase I demonstrated that this was not due to any additional sequences in the immediate upstream region. Likewise, analysis of Pac I, Sfi I and Not I digests (Figure 3) identified downstream fragments similar to the sizes predicted on GeneDB. These data are consistent the heterogeneity between 


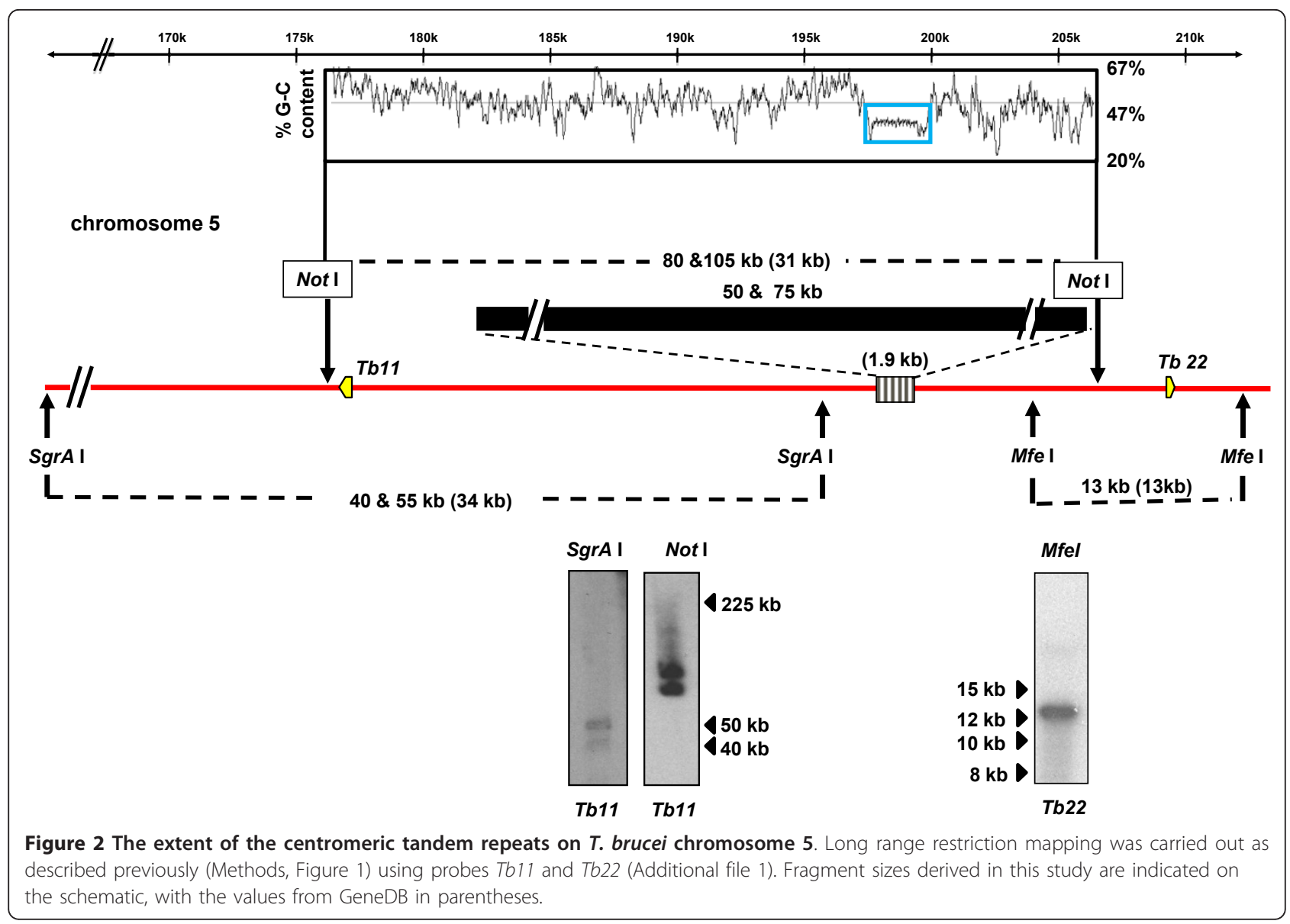

chromosomes, with the repeat arrays stretching over approximately $100 \mathrm{~kb}$ and $120 \mathrm{~kb}$.

\section{Discussion and Conclusions}

In eukaryotes, centromeric sequences are frequently organised as highly repetitive tandem arrays that stretch over extensive regions of chromosomal DNA. Their complete assembly has proven to be an intractable problem in most genome projects [32]. Where detailed analysis has been undertaken, considerable intrachromosomal size variation and sequence divergence has become apparent $[3,33]$. This arises from the acquisition of point mutations and high rates of unequal homologous recombination. When the T. brucei genome was initially completed [14], regions subsequently identified as centromeres, were characterised by the presence of $\sim 147$ bp repeat arrays predicted to extend over $2-8$ $\mathrm{kb}$ [18]. In each of the $8 \mathrm{~T}$. brucei chromosomes analysed here, our data suggest that these centromeric arrays are much larger (Table 1), varying from $20 \mathrm{~kb}$ (chromosome 1) to more than $100 \mathrm{~kb}$ (chromosome 7). We found a tendency for these regions to be more extensive in the larger chromosomes. This contrasts with $S$. pombe, where centromere length is inversely proportional to the chromosome length [34]. In addition, we also observed several instances of heterogeneity between chromosome homologues (Table 1). Using data available on TriTrypDB, there is no evidence for single nucleotide polymorphisms contributing to the generation of larger than expected restriction fragments containing the arrays. Although we cannot demonstrate unambiguously that the missing segments of centromeric DNA are constituted by tandem repeats, it would be unusual if extensive segments of non-repetitive sequences had been missed from the corresponding regions of each chromosome during the genome project.

The nature of the centromere-kinetochore complex in trypanosomes and the role that the $\sim 147$ bp repeats play in recruitment are two of the most intriguing unsolved questions in parasite biology. Although segregation in trypanosomes appears to be mediated by a conventional microtubule - kinetochore attachment, the number of kinetochores seems to be less than the number of chromosomes [35,36]. Trypanosomatids lack genes for the conserved "core" centromeric proteins, as well as the majority of other proteins involved in 


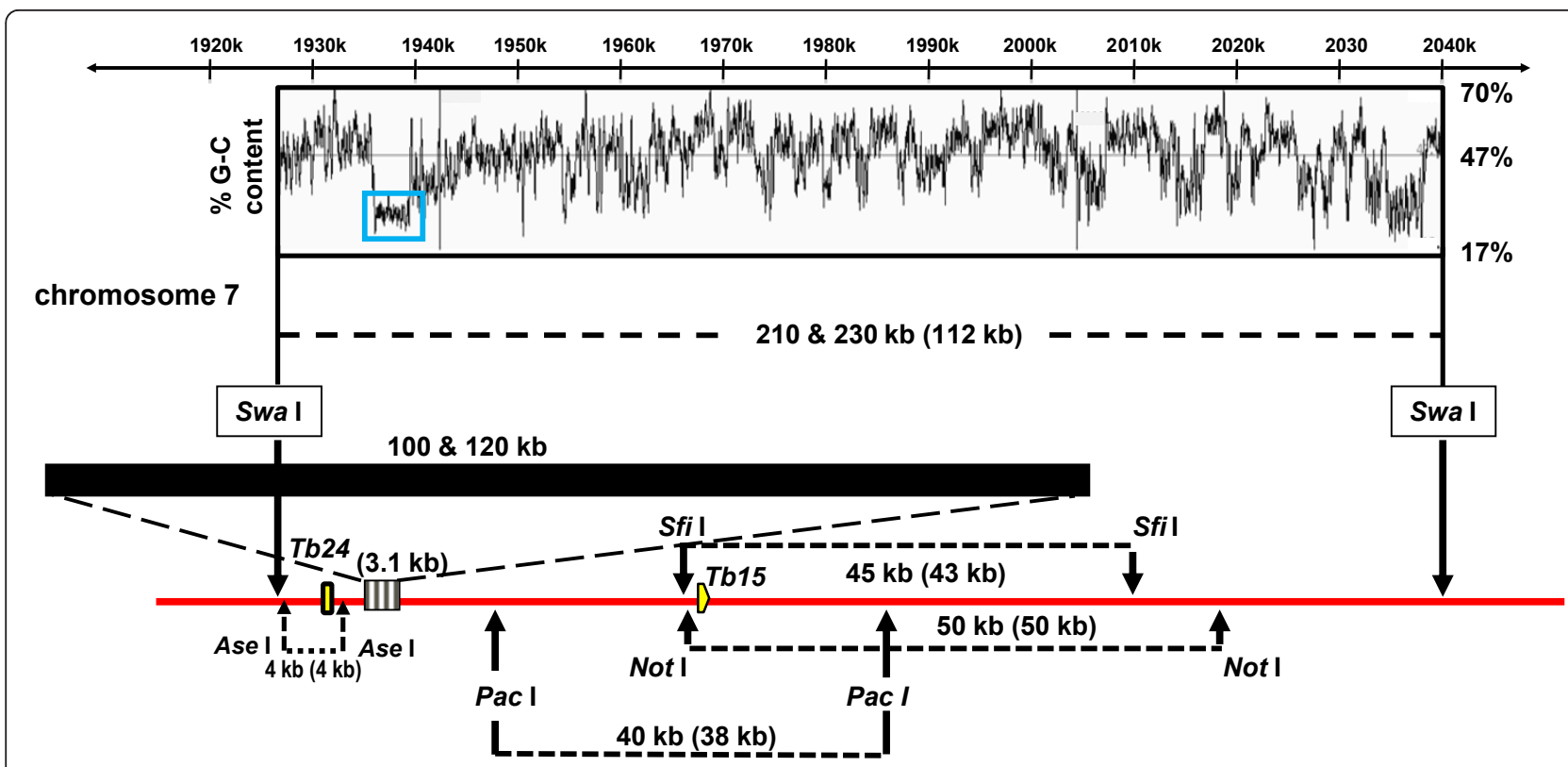

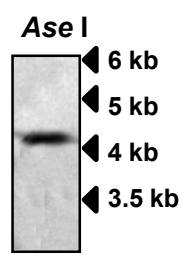

Tb24
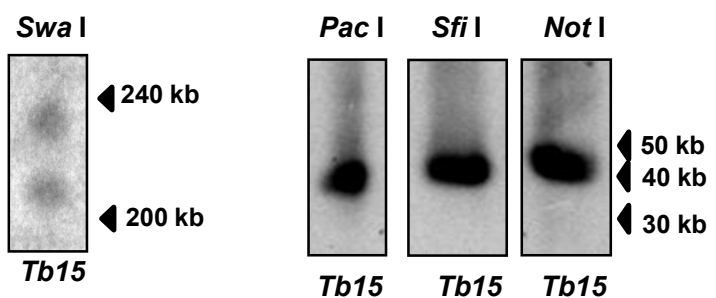

Figure 3 The extent of the centromeric tandem repeats on T. brucei chromosome 7. Analysis was carried out as described for Figures 1 and 2 using probes Tb24 and Tb15 (Additional file 1). Probe Tb24 was derived from a unique intergenic sequence. Fragment sizes derived in this study are indicated on the schematic, with the values from GeneDB in parentheses. The data suggest that the repeat arrays vary in length between chromosome homologues and stretch over at least100 kb (see also Table 1).

kinetochore assembly and function [14]. They are distinct in lacking an obvious orthologue of the variant histone CenH3, which replaces the canonical histone $\mathrm{H} 3$ at centromeres in other eukaryotes. In T. brucei, the only histone $\mathrm{H} 3$ variant identified is non-essential, enriched at telomeres, and lacks the extended loop I region or characteristic carboxyl terminal domain that are diagnostic of CenH3 [37-39] .

In other eukaryotes, CenH3 is essential for kinetochore assembly and functions as an epigenetic marker for centromere location [40]. By contrast, the centromeric repeat arrays with which they interact are not a pre-requisite. If normal centromere function is lost in some eukaryotes, neocentromeres can form in regions which lack these arrays, and once formed, the new location is stably inherited and specified epigentically by CenH3 binding $[3,40]$. Centromeric repeats then accumulate in these regions over time, where they may have a role in providing an environment that favours or promotes the formation of centromeric chromatin. Traditionally, centromeric heterochromatin had been considered transcriptionally quiescent. However several recent studies, initially in fission yeast, have highlighted an essential role for short interfering RNAs (siRNAs) derived from centromeric sequences in the formation of heterochromatin and centromere function $[41,42]$. Interestingly, a recent report has described a class of siRNAs derived from centromeric repeats in T. brucei, although their functional significance remains to be elucidated [43].

Our finding that the $\sim 147 \mathrm{bp}$ tandem repeats constitute a larger than expected component of T. bruce $i$ chromosomes provides an improved framework for investigating aspects of genome biology, including the determinants of centromere function. For example, the cell-cycle specific accumulation of topoisomerase-II at centromeres is required for regulated segregation of sister chromatids. Precise mapping of this decatenation activity onto T. brucei chromosomes was complicated by uncertainty over the size of the centromeric repeats arrays [18]. Likewise, analysis of chromatin immunoprecipitation experiments to assess the extent of histone 
modifications associated with centromeric domains would be difficult to interpret in the absence of a more accurate chromosome map. To date most studies on chromosome segregation have focused on mammals, insects, plants and fungi. Analysis of the situation in trypanosomes demonstrates both similarities and differences from the standard model. We have now shown that the organisation of centromeric DNA repeats in $T$. brucei conforms to the "regional" class, typical of higher eukaryotes. In contrast, the protein factors which mediate segregation are unknown, and by inference, must be highly divergent. Further studies aimed at uncovering the mechanisms involved are crucial to ensure that our understanding the chromosome segregation takes full account of eukaryotic diversity.

\section{Additional material}

Additional file 1: Details of probes and restriction sites used for mapping. A comprehensive list of DNA probes used for restriction mapping and the Artemis coordinates of the corresponding restriction enzymes.

Additional file 2: Analysis of restriction endonuclease mapping data for $T$. brucei chromosomes $1,2,4,6$, and 8 . Details on how the mapping data for those chromosomes not described in the text of the manuscript were interpreted.

Additional file 3: Delineation of the centromeric repeats on $T$. brucei chromosomes $1-8$ using long range restriction mapping. A complete collation of the mapping data from all of the T. brucei chromosomes analysed.

\section{List of Abbreviations}

CenH3: centromeric histone H3; CHEF(E): contour-clamped homogenous electric field (electrophoresis); siRNA, short interfering RNA; VSG: variant surface glycoprotein.

\section{Acknowledgements and funding}

JMK was supported by the UK Biotechnology and Biological Sciences Research Council (grant number BB/C501292/1) and the Wellcome Trust (grant number 084175). MCE was the recipient of a Colombian Research Council (COLCIENCIAS) and Universidad Nacional de Colombia scholarship. We acknowledge the work of our colleagues on the T. brucei Genome Project [ref. [14]] and thank Flora Logan (GeneDB; Wellcome Trust Sanger Institute) for assistance with accessing GeneDB sequence data and for useful discussions.

\section{Author details}

'Department of Infectious and Tropical Diseases, London School of Hygiene and Tropical Medicine, Keppel Street, London WC1E 7HT, UK. ' 2 Laboratorio de Parasitologia - Facultad de Medicina, Universidad Nacional de ColombiaSede, Bogota, Columbia. ${ }^{3}$ Laboratory of Cellular and Structural Biology, Rockefeller University, 1230 York Avenue, New York, NY 10065, USA.

\section{Authors' contributions}

MCE, designed the study, carried out the experiments and wrote the paper CB and SOO made the original observations, contributed to experimental design and commented on the manuscript. MCT contributed to experimental design and commented on the manuscript. JMK designed the study and wrote the paper. All authors read and approved the final manuscript.
Received: 21 October 2011 Accepted: 18 January 2012

Published: 18 January 2012

\section{References}

1. Sun X, Wahlstrom J, Karpen G: Molecular structure of a functional Drosophila centromere. Cell 1997, 91:1007-1019.

2. Sullivan BA, Blower MD, Karpen GH: Determining centromere identity: cyclical stories and forking paths. Nat Rev Genet 2001, 2:584-596.

3. Ma J, Wing RA, Bennetzen JL, Jackson SA: Plant centromere organization: a dynamic structure with conserved functions. Trends Genet 2007, 23:134-139.

4. Schueler MG, Higgins AW, Rudd MK, Gustashaw K, Willard HF: Genomic and genetic definition of a functional human centromere. Science 2001, 294:109-115.

5. Wong $\mathrm{LH}, \mathrm{Choo} \mathrm{KH}$ : Evolutionary dynamics of transposable elements at the centromere. Trends Genet 2004, 20:611-616.

6. Henikoff S, Ahmad K, Malik HS: The centromere paradox: Stable inheritance with rapidly evolving DNA. Science 2001, 293:1098-1102.

7. Malik HS, Henikoff S: Conflict begets complexity: the evolution of centromeres. Curr Opin Genet Dev 2002, 12:711-718.

8. Mehta GD, Agarwal MP, Ghosh SK: Centromere identity: a challenge to be faced. Mol Genet Genomics 2010, 284:75-94.

9. Ekwall K: Epigenetic control of centromere behaviour. Annu Rev Genet 2007, 41:63-81.

10. Steiner NC, Hahnenberger KM, Clarke L: Centromeres of the fission yeast Schizosaccharomyces pombe are highly variable genetic loci. Mol Cell Biol 1993, 13:4578-4587.

11. Pidoux AL, Allshire RC: Kinetochore and heterochromatin domains of the fission yeast centromere. Chrom Res 2004, 12:521-534.

12. Maddox PS, Oegema K, Desai A, Cheeseman IM: "Holo"er than thou: Chromosome segregation and kinetochore function in C. elegens. Chrom Res 2004, 12:641-653.

13. Campbell DA, Thomas S, Sturm NR: Transcription in kinetoplastid protozoa: why be normal? Microbes Infect 2003, 5:1231-1240.

14. Berriman M, Ghedin E, Hertz-Fowler C, Blandin G, Renauld H, Bartholomeu DC, Lennard NJ, Caler E, Hamlin NE, Haas B, et al: The genome of the African trypanosome Trypanosoma brucei. Science 2005, 309:416-422.

15. Gull K, Alsford S, Ersfield K: Segregation of minichromosomes in trypanosomes: implications for mitotic mechanisms. Trends Microbiol 1998, 6:319-323.

16. Foltz DR, Black BE, Bailey AO, Yates JR, Cleveland DW: The human CENP-A centromeric nucleosome-associated complex. Nature Cell Biol 2006, 8:458-469

17. Malik HS, Henikoff S: Phylogenomics of the nucleosome. Nat Struct Biol 2003, 10:882-889.

18. Obado SO, Bot C, Nilsson D, Andersson B, Kelly JM: Repetitive DNA is associated with centromeric domains in Trypanosoma brucei but not Trypanosoma cruzi. Genome Biol 2007, 8:R37.

19. Obado SO, Bot C, Echeverry MC, Bayona JC, Alvarez VE, Taylor MC, Kelly JM: Centromere-associated topoisomerase activity in bloodstream form Trypanosoma brucei. Nucl Acids Res 2011, 39:1023-1033.

20. Baumann C, Körner R, Hofmann K, Nigg EA: PICH, a centromere-associated SNF2 family ATPase, is regulated by Plk1 and required for spindle checkpoint. Cell 2007, 128:101-114.

21. Chan K-L, North PS, Hickson ID: BLM is required for faithful chromosome segregation and its localization defines a class of ultrafine anaphase bridges. EMBO J 2007, 26:3397-3409.

22. Spence JM, Critcher R, Ebersole TA, Valdivia MM, Earnshaw WC, Fukagawa T, Farr CJ: Co-localization of centromere activity, proteins and topoisomerase-II within a subdomain of the major human $\times$ alphasatellite array. EMBO J 2002, 21:5269-5280.

23. Jonstrup AT, Thomsen T, Wang Y, Knudsen BR, Koch J, Andersen AH: Hairpin structures formed by alpha satellite DNA of human centromeres are cleaved by human topoisomerase. Nucl Acids Res 2008, 36:6165-6174.

24. Obado SO, Taylor MC, Wilkinson SR, Bromley EV, Kelly JM: Functional mapping of a trypanosome centromere by chromosome fragmentation identifies a 16 kb GC-rich transcriptional "strand-switch" domain as a major feature. Genome Res 2005, 15:36-43. 
25. Kelly JM, McRobert L, Baker DA: Evidence on the chromosomal location of centromeric DNA in Plasmodium falciparum from etoposide-mediated topoisomerase-II cleavage. Proc Natl Acad Sci USA 2006, 103:6706-6711.

26. Iwanaga S, Khan SM, Kaneko I, Christodoulou Z, Newbold C, Yuda M, Janse CJ, Waters AP: Functional identification of the Plasmodium centromere and generation of a Plasmodium artificial chromosome. Cell Host Microbe 2010, 7:245-255.

27. Brooks CF, Francia ME, Gissot M, Croken MM, Kim K, Striepen B: Toxoplasma gondii sequesters centromeres to a specific nuclear region throughout the cell cycle. Proc Natl Acad Sci USA 2011, 108:3767-3772.

28. Brun R, Jenni L: A new semi-defined medium for Trypansoma brucei sspp. Acta Tropica 1977, , 34: 21-33.

29. Gibson WC, Miles MA: The karyotype and ploidy of Trypanosoma cruzi. EMBO J 1986, 5:1299-1305.

30. Kelly JM: Isolation of DNA and RNA from Leishmania. In Protocols in Molecular Parasitology. Volume 21. Edited by: Hyde JE. Humana Press, New Jersey; 1993:312-321, Methods in Molecular Biology (series editor Walker $J M)$.

31. Benson G: Tandem repeats finder: a program to analyze DNA sequences. Nucl Acid Res 1999, 27:573-580.

32. Alkan C, Cardone MF, Catacchio CR, Antonacci F, O'Brien SJ, Ryder OA, Purgato S, Zoli M, Della Valle G, Eichler EE, Ventura M: Genome-wide characterization of centromeric satellites from multiple mammalian genomes. Genome Res 2011, 21:137-145.

33. Plohl M, Luchetti A, Mestrović N, Mantovani B: Satellite DNAs between selfishness and functionality: structure, genomics and evolution of tandem repeats in centromeric (hetero)chromatin. Gene 2008, 409:72-82

34. Wood V, Gwilliam R, Rajandream M-A, Lyne M, Lyne R, Stewart A, Sgouros J, Peat N, Hayles J, Baker S, et al: The genome sequence of Schizosaccharomyces pombe. Nature 2002, 415:871-880.

35. Ogbadoyi E, Ersfeld K, Robinson D, Sherwin T, Gull K: Architecture of the Trypanosoma brucei nucleus during interphase and mitosis. Chromosoma 2000, 108:501-513.

36. Ersfeld K, Gull K: Partitioning of large and minichromosomes in Trypanosoma brucei. Science 1997, 276:611-614.

37. Alsford S, Horn D: Trypanosomatid histones. Mol Microbiol 2004, 53:365-372.

38. Lowell JE, Cross GA: A variant histone $\mathrm{H} 3$ is enriched at telomeres in Trypanosoma brucei. J Cell Sci 2004, 117:5937-5947.

39. Guse A, Carroll CW, Moree B, Fuller CJ, Straight AF: In vitro centromere and kinetochore assembly on defined chromatin templates. Nature 2011, 477:354-358.

40. Stimpson KM, Sullivan BA: Epigenomics of centromere assembly and function. Curr Opin Cell Biol 2010, 22:772-780.

41. Volpe TA, Kidner C, Hall IM, Teng G, Grewal SI, Martienssen RA: Regulation of heterochromatic silencing and histone $\mathrm{H} 3$ lysine-9 methylation by RNAi. Science 2002, 297:1833-1837.

42. Grewal SI: RNAi-dependent formation of heterochromatin and its diverse functions. Curr Opin Genet Dev 2010, 20:134-141

43. Patrick KL, Shi H, Kolev NG, Ersfeld K, Tschudi C, Ullu E: Distinct and overlapping roles for two Dicer-like proteins in the RNA interference pathways of the ancient eukaryote Trypanosoma brucei. Proc Natl Acad Sci USA 2009, 106:17933-17938.

44. Rutherford K, Parkhill J, Crook J, Horsnell T, Rice P, Rajandream MA, Barrell B: Artemis: sequence visualization and annotation. Bioinformatics 2000 , 16:944-945.

\section{Submit your next manuscript to BioMed Central and take full advantage of:}

- Convenient online submission

- Thorough peer review

- No space constraints or color figure charges

- Immediate publication on acceptance

- Inclusion in PubMed, CAS, Scopus and Google Scholar

- Research which is freely available for redistribution

Submit your manuscript at www.biomedcentral.com/submit
Biomed Central 